\title{
Micro-grid Monitoring System Based on Power Line Carrier of LonWorks
}

\author{
Ya Zhen ${ }^{1, a}$, Yanping Feng ${ }^{1, b}$ \\ ${ }^{1}$ Zhengzhou Technical College,China \\ aZhenya012001@163.com, b170298173@qq.com
}

Keywords: LonWorks; Kingview; Micro-grid; Monitoring System

\begin{abstract}
This paper introduces a design in developing of supervising system based on the LonWorks technology and Kingview. At first, the system combines Neuron chip with power supply controllers and switches as intelligent nodes to establish the network based on power line of LonWorks. In addition, through the software of LNS DDE Server, the system designs monitoring pictures with Kingview to show operation condition with real-time digital data for relevant operators to analysis and makes decisions. According to the results, it shows that the proposed monitor has the friendly manipulating interface, reliable performance, and high ability of expansibility.
\end{abstract}

\section{Introduction}

The micro grid is composed of load and a micro power, which can provide power and heat. Relative to the external large power grid, it is a single control unit, and meets users' requirements about power quality and supply reliability. Its energy management center sends commands to control the output power of the micro power, charging and discharging of the energy storage unit, start and stop of the load, as well as the real-time data display of running nodes. In order to ensure the stable operation of the micro grid, reliable communication between the energy management center and the nodes is a problem to solve[1].

The LonWorks control network based on power line carrier communication has been developed, where neuron chips are embedded into the micro power supply controllers and the load switches, making them intelligent nodes. The micro grid monitoring picture is designed with KingView. It is for real-time display and supporting the operators to complete micro power load distribution and load start-stop function, as well as providing related reports.

\section{Structure of the Micro Grid}

The structure of the micro grid is more flexible than traditional grids. Because it is connected with the public grid through the circuit breaker and points of common coupling (PCC), The load of the micro grid can obtain electricity from both the public grid and the micro grid[2].

\section{The Monitoring System Based on Power Line Carrier of LonWorks}

LonWorks, developed by an American company Echelon, is a kind of industrial control field bus for building automation. It consists of intelligent nodes, router and network management. Each node can control, collect and process data independently, while it communicates with other nodes and the workstation through the embedded LonTalk communication protocol, thus, to achieve the real intelligent distribution control[3].

a) System Structure

The micro grid system consists of three layers architecture, shown in Figure 1. The first layer is the field control layer, mainly the LON nodes, including the power supply controller, the storage controller and the load switch, respectively connected with the distributed power, the energy storage power and the load. The LON node is responsible for collecting the on-site information, including the current, voltage, phase of all the powers and the working state of the equipment, through the network variables. It is also responsible for storage, transmission and accept 
command from the top layers. So the LON node is the terminal of the micro grid system monitoring.

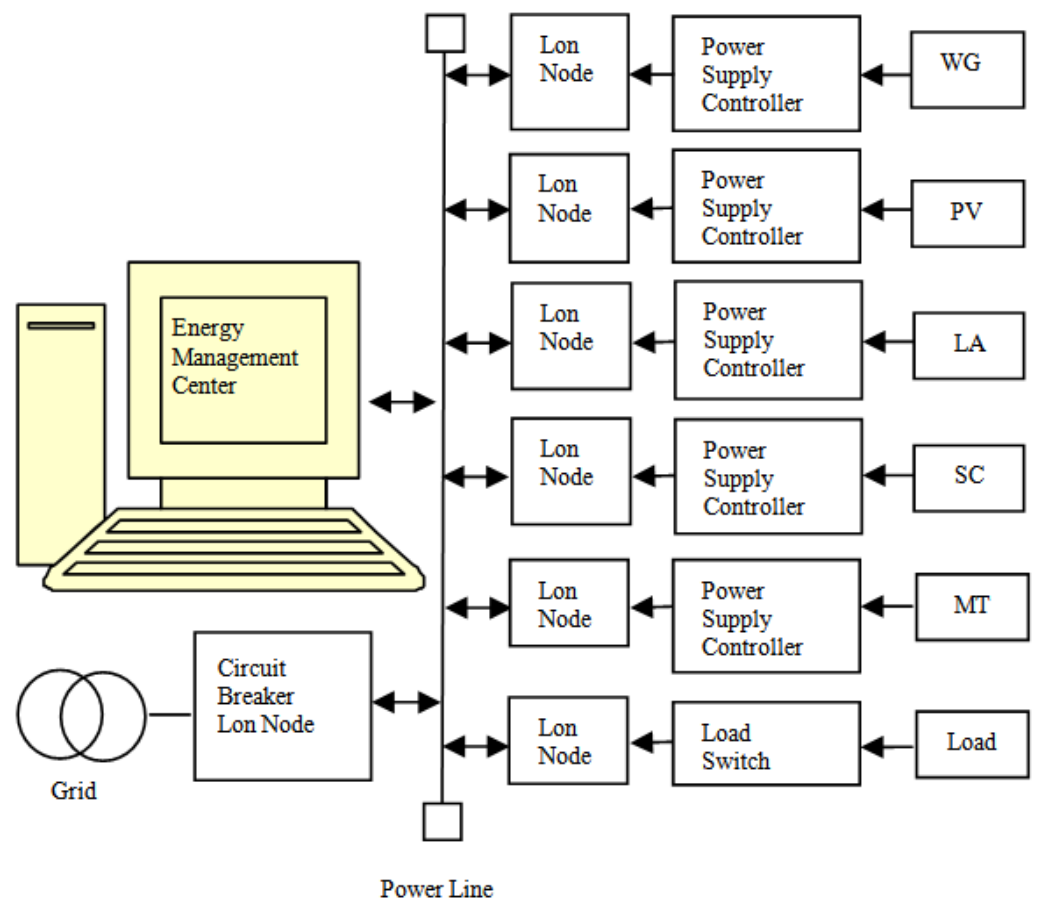

Figure 1. Architecture of Micro-grid.

The second layer is the network transport layer. Using the existing power line as the communication medium, FT3150 power line transceiver is responsible for the network communication between the LON node and the field bus system. And the LonWorks network is established through LonTalk protocol.

The third layer is the monitoring management layer, which is also the energy monitoring center. Remote monitoring is realized by the configuration software. Configuration monitoring software requests data from the LNS DDE Server through the driver software. There is data exchange between the LON nodes and the field control layer, thus monitoring the whole network. The energy management center provides the operation state of each basic unit to the operators through the man-machine interface. So it can execute the corresponding control strategy.

\section{Hardware design}

LonWorks equipment consists of the field device and the LON nodes. The former mainly includes the Power controller in the control system, the storage controller and the load switch. The latter is composed of a neuron chip, the corresponding peripheral circuit, and the power line transceiver. It can manage communication, as well as input, output and control function. The Design and development of intelligent nodes are divided into three types: the LON node for power control, the LON node for energy storage control and for the user load switch.

\section{Software design for intelligent node}

\section{Programming}

The program of the neuron chip is written with Neuron $C$ language in the NodeBuilder environment. Based on ANSIC and specially designed for the nerve chip, neuron is a high-level programming language with network communication and advanced hardware equipment monitoring extension. The development steps of the nodes are as follows.

1) Define the I/O object

Define corresponding variables according to different hardware devices. It is allowed to initialize the object, and set up its working parameters.

2) Define the timer object 
There are 15 timer objects to define at most to periodically execute some operations or delay when necessary.

3) Define network variables and display alarm

Both network variables and display the alarm can transmit information. Generally, network variables are often adopted.

4) Define the task

The task is to drive with Neuron C, which means reaction to some events.

5) Other user-defined functions

Put some commonly used functions in the header file for the program call to call. These user-defined functions can do some regular work.

\section{The establishment of the LonWorks network}

Each intelligent node is connected with the power line through a FT3150 transceiver. The working principle of the transceiver is shown in Figure 2. The control network is created by the LonMarker software, which can design and allocate the LonWorks control network. It contains a powerful network service structure and a Microsoft Visio interface based on LNS network system. After successful compilation, each Lon node can combine the function blocks to realize logical connection.

\section{Monitoring software design}

\section{Kingview}

Kingview supports integrated systems, composed of industry standard software and hardware platform, instead of a traditional closed system. It provides a main interface of resource management type, as well as a scripting language with Chinese characters as keywords. The micro grid monitoring pictures can be obtained conveniently because of its graphical editing feature. Kingview also has alarm, real-time trend curve display and other functions.

\section{Data communication}

Data communication refers to the communication between the monitor program and the LonWorks network, including the hardware connection and the communication driver configuration. The LNS DDE Server is for the configuration properties and the exchange of network variables between the LonWorks nodes and Windows client application. And it can be applied to any application with a standard Windows DDE interface. Therefore, DDE communication mode is adopted in the configuration of communication drive. The monitoring program can exchange data with the LonWorks intelligent nodes through the LNS DDE Server.

\section{The database establishment}

The real-time database is a bridge between the management Server and the LonWorks nodes, which can realize real-time transmission and make the configuration software to display. The database variables are mainly the system variables and user-defined variables.

The I/O variable is for dynamical data exchange between the Configuration software and the LonWorks nodes. For example, the switch state of Load 1 corresponds to nvo_switch[0], and the output power command of the energy storage controller corresponds to energy nvi_energystorage[0]. Once the I/O variable changes, this new value will be written into the application automatically; When the value in the application changes, Kingview will change correspondingly.

\section{The monitoring interface}

The wiring diagram is shown in the main interface, shown in Figure 2. Clicking the button on the menu bar can switch pictures like the historical trend curves and the real-time data reports. The real time data reports can help the operators obtain real-time micro grid data. 


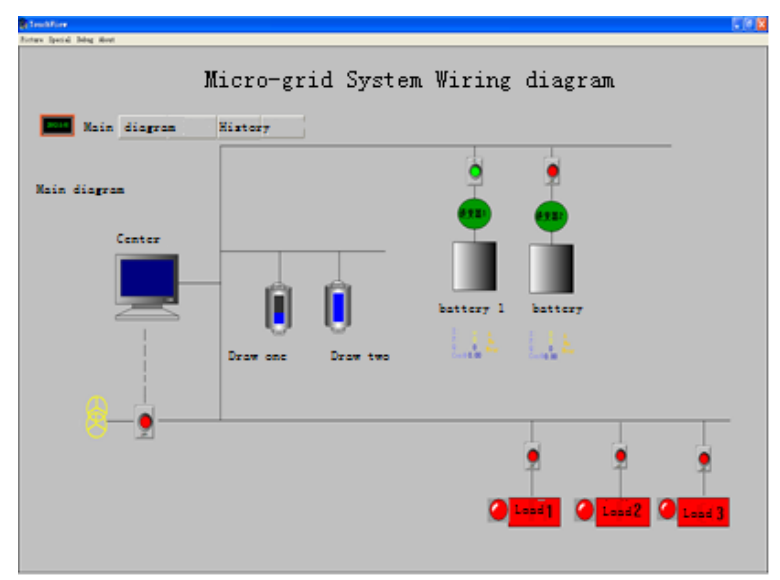

Figure 2. Wiring diagram

\section{The program command language}

Program, which runs automatically when the monitoring software is on, is the program command language. Variables used in the language are defined in the engineering data dictionary. The control strategy in both island mode and grid mode is programmed in the command window. The monitoring software will adjust the inverter and the load switch according to different control strategy.

\section{Conclusion}

The paper has developed a micro grid monitoring system based on power line carrier of Lonworks and Kingview. The monitoring system has the following features:

1) The communication medium is the power line with simple wiring to reduce the cost of network construction;

2) Each control unit is relatively independent with high efficiency of the network;

3) The monitoring system is portable, extensible with strong networking ability.

The experimental results show that, the monitoring system has friendly interfaces, reliable performance and strong expansion capability.

\section{References}

[1] SU Ling, Zhang Jian-hua, et al. Study on some key problems and technique related to microgrid[J]. Power System Protection and Control, 2010,38(19):235-239.

[2] SHENG Kun, KONG Li, et al. A survey on research of micro grid -a new power system, Relay, 2007,35(12):75-81.

[3] YANG Yu-hong. LON network control technology and its application [M]. Xi 'an university of electronic science and technology press, 1999. 